\title{
Fourier-transform Spectroscopic Technique for Multi-spectral Nonlinear Microscopy Using a 5-fs Broadband Light Source
}

\author{
Keisuke ISOBE, ${ }^{1}$ Akira SUDA, ${ }^{1}$ Masahiro TANAKA,${ }^{2}$ Fumihiko KANNARI, ${ }^{2}$ Hiroyuki KAWANO,${ }^{3}$ \\ Hideaki MIZUNO, ${ }^{3}$ Atsushi MIYAWAKI,${ }^{3}$ and Katsumi MIDORIKAWA ${ }^{1}$ \\ ${ }^{1}$ Laser Technology Laboratory, RIKEN, 2-1 Hirosawa, Wako-city, Saitama 351-0198 \\ ${ }^{2}$ Department of Electronics and Electrical Engineering, Keio University, \\ 3-14-1 Hiyoshi, Kohoku-ku, Yokohama, 223-8522 \\ ${ }^{3}$ Laboratory for Cell Function Dynamics, Brain Science Institute, RIKEN, 2-1 Hirosawa, Wako-city, Saitama 351-0198
}

(Received January 18, 2008)

\begin{abstract}
We propose nonlinear Fourier-transform spectroscopy using a broadband pulse, which can discriminate the origin in Four-wave mixing (FWM) process. By taking account of the function of a sample in the third-order interferometric autocorrelation (IAC) signal, we theoretically analyze FWM-FTS using a broadband pulse. The discrimination of the origin is achieved by taking advantage of the FWM power spectrum obtained by the Fourier-transform of the IAC signal. In the FWM power spectrum, there are frequency components, which indicate the effects of the two-photon electronic resonance and the Raman resonance. By focusing into the frequency components, the origin in FWM process is identified. Based on theoretical analysis, we experimentally demonstrate nonlinear FTS employing the tightly focused pulse with a pulse duration of 5 fs and measure the Raman and two-photon electronic spectra in FWM process.
\end{abstract}

Key Words: Four-wave mixing, Nonlinear optical spectroscopy, Fourier-transform spectroscopy

\section{Introduction}

Four-wave mixing (FWM) microscopy has become an important tool for the investigation of biological phenomena $1-3)$ because of its capability to produce three-dimensional images without fluorescent labelling. ${ }^{4-6)}$ In FWM microscopy, molecules are identified by taking advantage of the spectral information in FWM process. However, it has been difficult to distinguish the origin of the FWM signal because the wavelength of the FWM signal does not depend on the contributions from the Raman resonance, electronic resonance and nonresonance.

In this paper, we propose a novel technique for discriminating the origin in FWM process. This technique is based on nonlinear Fourier-transform spectroscopy (FTS) ${ }^{7-9)}$ combined with a broadband pulse. It has been reported that nonlinear FTS can be applied to Raman-resonance FWM, ${ }^{7)}$ two-photon excited fluorescence (TPEF) ${ }^{8)}$ and sum-frequency generation. ${ }^{9)}$ The power spectrum is obtained by the Fourier-transformation of an interferometric autocorrelation (IAC) signal. Thus, the spectral resolution is determined not by the spectral width of excitation light source by but the inverse of maximum delay time between the excitation pulses. Therefore, high spectral resolution can be achieved even though a single broadband pulse laser, which produces sub-10-fs pulses and enables simultaneous measurement over a wide range of the spectrum, is used. In the case of the use of broadband pulses, it would be advantageous that the pulses are tightly focused with a high-numerical-aperture objective lens because the phase matching condition in FWM process is largely relaxed due to the wide cone of wave vectors and the short interaction length. However, it is difficult to generate the tightly focused pulse of which pulse duration is shorter than 10 fs because lens chromaticity and spherical aberration lead not only to the spatial distortion but also to an unfavorable pulse broadening at larger apertures ${ }^{10)}$ and the dispersion of a high-numerical-aperture objective becomes serious. In spite of some efforts on nonlinear FTS, this technique has not been used for the measurement of the effect of the two-photon electronic (TPE) resonance in FWM process. A possible reason is that it is hard to generate a tightly focused pulse with a pulse duration of sub- $10 \mathrm{fs}$.

By taking account of the third-order IAC signal including the response function of a sample, we theoretically analyze FWM-FTS using a broadband pulse. When a broadband pulse is employed as an excitation light source, the FWM power spectrum shows not only the Raman resonance but also the TPE resonance. By comparison of the FWM power spectrum from a resonant sample with that from a nonresonant reference sample, the origin in FWM process is identified. In the experiment, by generating a tightly focused pulse with a pulse duration of 5-fs, we demonstrate that FWM-FTS can be applied to the measurement of the effects of not only Raman resonance of but also TPE resonance and achieve the discrimination of the origin in FWM process.

\section{Theory}

In FWM process, a photon with a frequency of $2 \omega_{1}-\omega_{2}$ is generated by the interaction between the medium and the photons with frequencies of $\omega_{1}$ and $\omega_{2}$. In the two-photon resonance FWM process, there are the electronic resonance 


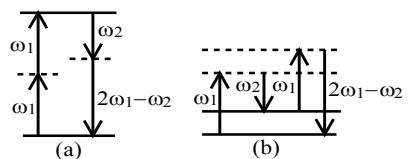

Fig. 1 Energy diagram for FWM processes under the TPE resonance (a) and the Raman resonance (b).

and Raman resonance (see Fig. 1). The frequency of the FWM signal is $2 \omega_{1}-\omega_{2}$ for both cases of the electronic resonance and the Raman resonance. In order to discriminate the signal under the electronic resonance from that under the Raman resonance, it has been necessary to measure the dependence of the signal intensity on the pump frequency $\left(2 \omega_{1}\right)$ and on the frequency difference $\left(\omega_{1}-\omega_{2}\right)$ so far. In this section, we show that the origin of the FWM signal can be distinguished by taking advantage of the FWM power spectrum obtained by FTS technique.

We show the principle of FWM-FTS combined with a single broadband pulse laser. FWM-FTS is based on the IAC measurement. The third-order IAC signal $S_{3}(\tau)$ is given by

$$
S_{3}(\tau)=\int_{-\infty}^{\infty}\left|P^{(3)}(t, \tau)\right|^{2} d t
$$

where $\tau$ is the delay time between the broadband pulses and $P^{(3)}(t, \tau)$ is the nonlinear polarization. We first analyze the FWM power spectrum obtained by Fourier-transform of third-order IAC signal by FWM process under the TPE resonance. The polarization in FWM process under the TPE resonance is described by

$$
P_{\mathrm{TPE}}^{(3)}(t, \tau)=E_{2}^{*}(t, \tau) \int R^{(\mathrm{TPE})}\left(t-t_{1}\right) E_{1}\left(t_{1}, \tau\right)^{2} d t_{1},
$$

where $E_{\mathrm{i}}(t, \tau)=\left[A_{\mathrm{i}}(t)+A_{\mathrm{i}}(t-\tau) \exp \left(-\mathrm{i} \omega_{\mathrm{i}} \tau\right)\right] \exp \left(\mathrm{i} \omega_{\mathrm{i}} t\right),(\mathrm{i}=1,2)$. Here, we define the electric fields as $E_{\mathrm{i}}(t)=A_{\mathrm{i}}(t) \exp \left(\mathrm{i} \omega_{\mathrm{i}} t\right)$, where $A_{\mathrm{i}}(t)$ is slowly-varying amplitude. The absolute square of $P_{\mathrm{TPE}}(t, \tau)$ is expressed as

$$
\begin{aligned}
\left|P_{\mathrm{TPE}}^{(3)}(t, \tau)\right|^{2}= & B_{0}(t, \tau)+B_{1}(t, \tau) \exp \left(i \omega_{2} \tau\right) \\
& +B_{2}(t, \tau) \exp \left(i \omega_{1} \tau\right)+B_{3}(t, \tau) \exp \left\{i\left(\omega_{1}+\omega_{2}\right) \tau\right\} \\
& +B_{4}(t, \tau) \exp \left\{i\left(\omega_{1}-\omega_{2}\right) \tau\right\}+B_{5}(t, \tau) \exp \left(i 2 \omega_{1} \tau\right) \\
& +B_{6}(t, \tau) \exp \left\{i\left(2 \omega_{1}-\omega_{2}\right) \tau\right\} \\
& +B_{7}(t, \tau) \exp \left\{i\left(2 \omega_{1}+\omega_{2}\right) \tau\right\} \\
& +C C
\end{aligned}
$$

where, CC indicates the complex conjugate. $B_{\mathrm{i}}(t, \tau)$ is the function of $A_{\mathrm{i}}(t), A_{\mathrm{i}}(t-\tau), R^{(\mathrm{TPE})}, \omega_{\mathrm{i}}$, and $\tau$. From eq. (3) we find that the FWM-IAC signal by the TPE resonance includes the fringe components around frequencies of $0, \omega_{1}, \omega_{2}, \omega_{1}-\omega_{2}$, $\omega_{1}+\omega_{2}, 2 \omega_{1}, 2 \omega_{1}-\omega_{2}$ and $2 \omega_{1}+\omega_{2}$. We focus on the fringe component around a frequency of $2 \omega_{1}$. The FWM power spectrum around a frequency of $2 \omega_{1}$ is given by

$$
\begin{aligned}
& S_{2 \omega_{1}}^{(\mathrm{TPE})}(\Omega)=\widetilde{R}^{(\mathrm{TPE})^{*}}(\Omega) \widetilde{A}_{11}^{(2)^{*}}\left(\Omega-2 \omega_{1}\right) \int \widetilde{A}_{22}^{(2+)}\left(\Omega-\Omega_{1}\right) \widetilde{R}^{(\mathrm{TPE})}\left(\Omega_{1}\right) \widetilde{A}_{11}^{(2)}\left(\Omega_{1}-2 \omega_{1}\right) d \Omega_{1} \\
& +\widetilde{R}^{(\mathrm{TPE})}(\Omega) \tilde{A}_{11}^{(2)}\left(\Omega-2 \omega_{1}\right)\left[\left[\tilde{A}_{22}^{(2 f)}\left(\Omega-\Omega_{1}\right) \widetilde{R}^{(\mathrm{TPE})}\left(\Omega_{1}\right) \tilde{A}_{11}^{(2)}\left(\Omega_{1}-2 \omega_{1}\right) d \Omega_{1}\right],\right.
\end{aligned}
$$

where

$$
\tilde{A}_{\mathrm{ij}}^{(2 \#)}(\omega)=\int \tilde{A_{\mathrm{i}}}\left(\Omega_{1}\right) \tilde{A}_{\mathrm{j}}^{*}\left(\Omega_{1}-\omega\right) \mathrm{d} \Omega_{1}
$$

$\tilde{A}_{\mathrm{ij}}{ }^{(2)}(\omega)=\int \tilde{A_{\mathrm{i}}}\left(\Omega_{1}\right) \tilde{A}_{\mathrm{j}}\left(\omega-\Omega_{1}\right) \mathrm{d} \Omega_{1}$. For the case of the sample whose spectral bandwidth of absorption is narrow such as an atomic vapor and fluorophores at extremely low temperatures, we can assume that $R^{(\mathrm{TPE})}(\Omega)$ is $\delta\left(\Omega-\Omega_{0}\right)$, then we obtain

$$
\begin{aligned}
S_{2 \omega_{1}}^{(\mathrm{TPE})}(\Omega) \approx & \delta\left(\Omega-\Omega_{0}\right) \tilde{A}_{22}^{(2 \sharp)}\left(\Omega-\Omega_{0}\right)\left|\widetilde{A}_{11}^{(2)}\left(\Omega_{0}-2 \omega_{1}\right)\right|^{2} \\
& +\delta\left(\Omega-\Omega_{0}\right) \widetilde{A}_{22}^{(2 \#)^{*}}\left(\Omega-\Omega_{0}\right)\left|\widetilde{A}_{11}^{(2)}\left(\Omega_{0}-2 \omega_{1}\right)\right|^{2} .
\end{aligned}
$$

In the FWM power spectrum around a frequency of $2 \omega_{1}$, the only resonant frequency component remains in existence. Thus the Fourier spectrum of the response function can be obtained.

On the other hand, if the spectral bandwidth of $R^{(\mathrm{TPE})}$ is broader than that of $\tilde{A}_{11}{ }^{(2)}\left(\Omega-2 \omega_{1}\right)$, we can regard eq. (4) as

$$
\begin{aligned}
S_{2 \omega_{1}}^{(\mathrm{TPE})}(\Omega) \approx & \widetilde{R}^{(\mathrm{TPE})^{*}}(\Omega) \tilde{A}_{11}^{(2) *}\left(\Omega-2 \omega_{1}\right) \int \widetilde{A}_{22}^{(2 \#)}\left(\Omega-\Omega_{1}\right) \widetilde{A}_{11}^{(2)}\left(\Omega_{1}-2 \omega_{1}\right) d \Omega_{1} \\
& +\widetilde{R}^{(\mathrm{TPE})}(\Omega) \widetilde{A}_{11}^{(2)}\left(\Omega-2 \omega_{1}\right)\left[\int \widetilde{A}_{22}^{(2 \#)}\left(\Omega-\Omega_{1}\right) \widetilde{A}_{11}^{(2)}\left(\Omega_{1}-2 \omega_{1}\right) d \Omega_{1}\right] .
\end{aligned}
$$

$\tilde{A}_{11}{ }^{(2)}\left(\Omega-2 \omega_{1}\right)\left[\int \tilde{A}_{22}{ }^{(2 \#)}\left(\Omega-\Omega_{1}\right) \tilde{A}_{22}{ }^{(2)}\left(\Omega_{1}-2 \omega_{1}\right) \mathrm{d} \Omega_{1}\right]^{*} \quad$ can $\quad$ be derived from the IAC signal by FWM in a nonresonant sample. Therefore we can obtain the response function of a resonant sample by measuring IAC signals by FWM from the TPE resonant sample and a nonresonant reference sample. We also consider the FWM power spectrum obtained by Fourier-transform of IAC signal by FWM process under the Raman resonance. The polarization in FWM process under the Raman resonance is described by

$$
P_{\text {Raman }}^{(3)}(t, \tau)=E_{1}(t, \tau) \int R^{(\text {Raman })}\left(t-t_{1}\right) E_{1}\left(t_{1}, \tau\right) E_{2}^{*}\left(t_{1}, \tau\right) d t_{1} .
$$

By calculating the absolute square of $P_{\text {Raman }}(t, \tau)$, we found that the FWM-IAC signal under the Raman resonance is also composed of the fringe components around frequencies of 0 , $\omega_{1}, \omega_{2}, \omega_{1}-\omega_{2}, \omega_{1}+\omega_{2}, 2 \omega_{1}, 2 \omega_{1}-\omega_{2}$ and $2 \omega_{1}+\omega_{2}$. The FWM power spectrum around a frequency of $\omega_{1}-\omega_{2}$ is expressed by

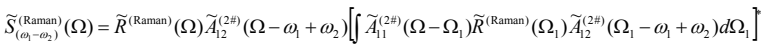

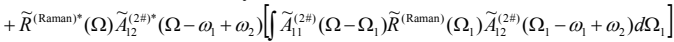

$$
\begin{aligned}
& +\int d \Omega_{1} \widetilde{R}^{\text {(Ramann })}\left(\Omega_{1}\right) A_{1}^{*}\left(\Omega_{1}\right) \tilde{A}_{1}^{*}\left(\Omega_{1}-\Omega\right) \widetilde{A}_{2}\left(\Omega_{1}-\Omega+\omega_{1}-\omega_{2}\right) \int \widetilde{A}_{1}\left(\Omega_{2}\right) \widetilde{G}_{1}\left(\Omega_{1}-\Omega_{2}\right) d \Omega_{2} \\
& +\int d \Omega_{1} \widetilde{R}^{\text {(Ramana) }}\left(\Omega_{1}\right) A_{1}\left(\Omega_{1}\right) \tilde{A}_{1}\left(\Omega_{1}-\Omega\right) \tilde{A}_{2}^{*}\left(\Omega_{1}-\Omega+\omega_{1}-\omega_{2}\right) \int \widetilde{A}_{1}^{*}\left(\Omega_{2}\right) \widetilde{G}_{1}^{*}\left(\Omega_{1}-\Omega_{2}\right) d \Omega_{2}
\end{aligned}
$$

If the delay time $\tau$ is longer than the pulse duration and is shorter than the Raman coherence lifetime, IAC signal under the Raman condition is expressed by

$$
\begin{array}{rl}
S^{(\text {Ramanff })}(\tau)=\int d & d\left\lfloor\left|A_{1}(t)\right|^{2}\left|G_{1}(t)\right|^{2}+\left|A_{1}(t-\tau)\right|^{2}\left|G_{2}(t, \tau)\right|^{2}\right. \\
& +\left[\left|A_{1}(t)\right|^{2}+\left|A_{1}(t-\tau)\right|^{2}\right] G_{1}(t) G_{2}^{*}(t, \tau) \exp \left\{i\left(\omega_{1}-\omega_{2}\right) \tau\right\} \\
& \left.+\left[\left|A_{1}(t)\right|^{2}+\left|A_{1}(t-\tau)\right|^{2}\right] G_{1}^{*}(t) G_{2}(t, \tau) \exp \left\{-i\left(\omega_{1}-\omega_{2}\right) \tau\right\}\right],
\end{array}
$$

where, $G_{\mathrm{i}}$ is the function of $A_{\mathrm{i}}(t), A_{\mathrm{i}}(t-\tau), R^{(\mathrm{Raman})}, \omega_{\mathrm{i}}$, and $\tau$. Equation (9) indicates that when the Raman coherence is not induced, the FWM signal is not generated by the interaction between the two pulses that are not temporally overlapped. Even though the nonresonance FWM signal and/or the electronic resonance FWM signal are mixed into the Raman resonance FWM signal, the FWM-IAC signal shown in eq. (9) can be obtained in the delay time that is longer than the pulse duration. From eq. (9) the FWM-IAC signal under the Raman resonance condition is composed of only the fringe components around frequencies of 0 and $\omega_{1}-\omega_{2}$. The FWM power spectrum around a frequency of $\omega_{1}-\omega_{2}$ is obtained by

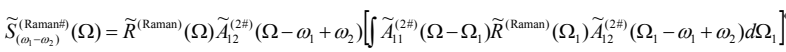

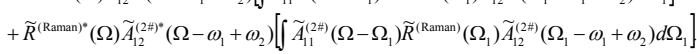

Since the Raman bandwidth is typically on the order of 10 
$\mathrm{cm}^{-1}$, the bandwidth of $\tilde{A}_{12}{ }^{(2 \#)}(\Omega)$ in a sub-10-fs pulse is much broader than the Raman bandwidth. Therefore, we can regard $R^{(\text {Raman })}(\Omega)$ as $\delta\left(\Omega-\Omega_{0}\right)$ and simplify eq. $(10)$ as

$$
\begin{aligned}
\widetilde{S}_{\left(\omega_{1}-\omega_{2}\right)}^{(\text {Ramant) }}(\Omega) \approx & \delta\left(\Omega-\Omega_{0}\right) \widetilde{A}_{11}^{(2 *)}\left(\Omega-\Omega_{0}\right)\left|\widetilde{A}_{12}^{(2 *)}\left(\Omega_{0}-\omega_{1}+\omega_{2}\right)\right|^{2} \\
& +\delta\left(\Omega-\Omega_{0}\right) \widetilde{A}_{11}^{(2 \neq * *}\left(\Omega-\Omega_{0}\right)\left|\widetilde{A}_{12}^{(2 *)}\left(\Omega_{0}-\omega_{1}+\omega_{2}\right)\right|^{2} .
\end{aligned}
$$

Equation (11) indicates the Fourier spectrum of the response function. By measuring the FWM-IAC signal in the delay time range that is longer than the pulse duration and is shorter than the Raman coherence time, we can obtain the response function of a Raman resonance sample. It should be noted that the origin of the FWM signal can be discriminated by focusing on the FWM power spectrum around frequencies of $2 \omega_{1}$ or $\omega_{1}-\omega_{2}$.

\section{Experiments}

\subsection{Experimental setup}

A schematic of the experimental setup is shown in Fig. 2. As a broadband light source, we used a Ti:sapphire laser (Nanolayers, Venteon) operating at a repetition rate of $80 \mathrm{MHz}$. The laser spectrum ranged from $660 \mathrm{~nm}$ to $1100 \mathrm{~nm}$. In order to compensate for the dispersion of optical components before the focal point of microscope objectives (OB1 and OB2), the laser pulse passed through a fused silica prism pair and a grating-pair-formed pulse shaper with a liquid-crystal spatial light modulator (SLM) (Cambridge Research \& Instrumentation, Inc., LC-SLM-128) placed at a Fourier plane. At the SLM, the excitation spectrum was limited to the region from $660 \mathrm{~nm}$ to $1000 \mathrm{~nm}$ for improving the spectral resolution of the spectral phase modulation. The prechirped laser pulse was sent into a Michelson interferometer. One half and the other half of the output were focused into a sample and a nonresonant reference sample, respectively, by microscope objectives (OBs) (Olympus Corporation, UPLSAPO40×, NA $0.9)$ whose chromatic aberration and spherical aberration were optimized for the spectral region from visible to near-infrared. The each resultant signal was detected with a photomultiplier tube (PMT) (Hamamatsu Photonics, R7400U-6) after the excitation light was eliminated by an excitation-light cut filter (Showa Optronics Co., Ltd., Broadband dichroic filter for Ti:sapphire laser). IAC signals were obtained by scanning a piezoelectric transducer stage with a step size of $5 \mathrm{~nm}$, which corresponds to a delay time of 33 as. The maximum delay time

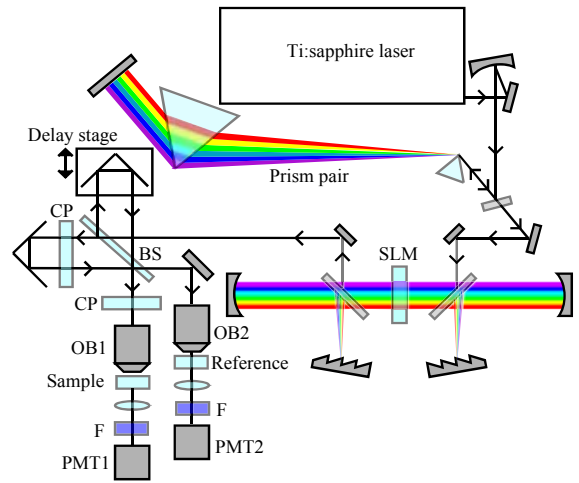

Fig. 2 Experimental setup of nonlinear Fourier-transform spectroscopy. BS: beam splitter, OB: objective lens, CP: compensation plate, PMT: photomultiplier tube. was $533 \mathrm{fs}$.

We generated Fourier-transform limited (FTL) pulse with a pulse duration of $5.3 \mathrm{fs}$ at the focal point of the objectives with the SLM phase-mask obtained by simulated annealing (SA) method. In the SA method, the pulse from one arm of the interferometer was focused onto a $10-\mu \mathrm{m}$ thickness beta-barium borate (BBO) crystal and an SLM phase-mask to maximize the resultant SHG was searched. The optimized pulses were measured by the SHG-IAC method.

To demonstrate identification of the origin of the FWM signal, we measured the FWM-IAC signals that were due to the Raman or TPE contribution. Taking advantage of the broadband spectrum, we utilized the intra-pulse FWM effect induced by electric field components with frequencies of $\omega_{1}$ and $\omega_{2}$ in the single broadband pulse. The wavelength of the FWM signal was around $580 \mathrm{~nm}$. The short wavelength components around $700 \mathrm{~nm}$ acted as two pump pulses with a frequency of $\omega_{1}$, while the long wavelength components around $900 \mathrm{~nm}$ served as a pulse with a frequency of $\omega_{2}$.

3.2 Measurement of Raman spectrum in four-wave mixing In order to confirm the Raman resonance effect in the FWM-IAC measurement, we used organic solvent as the sample with the Raman resonance. Figure 3(a) shows the FWM-IAC signal obtained from acetone. The Fourier-transform of the FWM-IAC signal is shown in Fig. 3(b). We found that in the Fourier spectrum, there were frequency components of $0, \omega_{1}-\omega_{2}, \omega_{1}, \omega_{2}, 2 \omega_{1}-\omega_{2}, \omega_{1}+\omega_{2}, 2 \omega_{1}$ and $2 \omega_{1}+\omega_{2}$. We focused only on the frequency component of $\omega_{1}-\omega_{2}$. The blue line in Figure 3(c) shows the magnified view of the frequency component around $\omega_{1}-\omega_{2}$ in Fig. 3(b). To compare the FWM power spectrum obtained from acetone
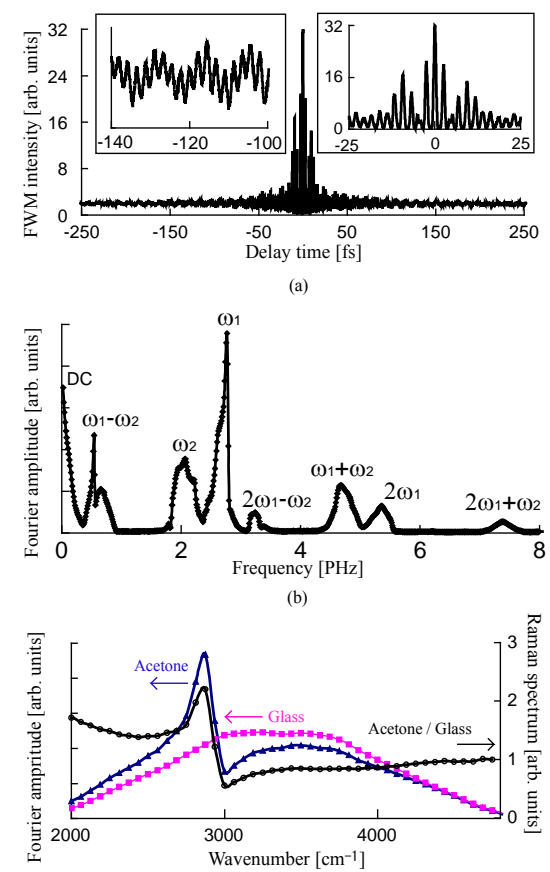

Fig. 3 (a) FWM-IAC signal obtained from acetone. (b) FWM power spectrum obtained by Fourier-transform of FWM-IAC signal shown in (a). (c) FWM power spectra around a frequency of $\omega_{1}-\omega_{2}$ and Raman spectrum. 
with that from a nonresonant sample, we measured the FWM-IAC signal from a cover glass. The FWM power spectrum of a cover glass is shown by the red line in Fig. 3(c). We found that the frequency components around a wavenumber of $2937 \mathrm{~cm}^{-1}$ were enhanced. This wavenumber is identified with the $\mathrm{CH}$ stretching vibration. The black line in Fig. 3(c) indicates the FWM Raman spectrum obtained by dividing the FWM power spectrum of acetone by that of the cover glass. It must be emphasized that the measurable bandwidth at a time is $3000 \mathrm{~cm}^{-1}$. This FWM Raman spectrum included the nonresonant contribution that is uniform against a frequency. To remove the effect of the instantaneous response that is due to the electronic contribution, we measured the FWM-IAC signal in the delay time range that was longer than the pulse duration. The sample was ethanol. The FWM-IAC signal and its Fourier-transform show in Figs. 4(a) and (b), respectively. We found that this power spectrum did not include the electronic contribution. It should be noted that the pure Raman spectrum can be obtained without a nonresonant reference sample.

3.3 Measurement of TPE spectrum in four-wave mixing

Next, we measured the FWM-IAC signal that was due to TPE resonance contribution. The sample was an anthracene powder whose resonant frequency was around $350 \mathrm{~nm}$. Under the TPE resonance condition, a TPEF was also generated from the anthracene powder. The bandpass filter (Semrock, Inc., FF01-593/40) was used for eliminating the TPEF. We used the quartz as the nonresonant reference sample. To confirm the TPE resonance effect, we focused on the frequency components around a frequency of $2 \omega_{1}$. This frequency corresponded to a wavelength of $350 \mathrm{~nm}$. The FWM power spectra from the anthracene powder and the quartz are shown by the blue and red lines in Fig. 5, respectively. The black line in Fig. 5 indicates the TPE spectrum that was obtained by dividing the power spectrum from the anthracene by that from the quartz. The wavelength dependence by the TPE resonance appeared clearly. By using a 5-fs broadband pulse, we could
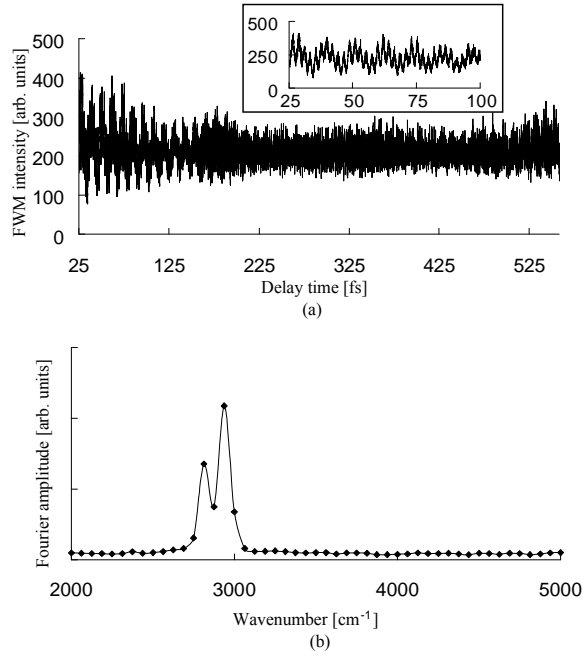

Fig. 4 (a) FWM-IAC signal with the delay time that is longer than pulse duration and is shorter than Raman coherence time. (b) FWM power spectrum obtained by Fourier-transform of FWM-IAC signal shown in (a).

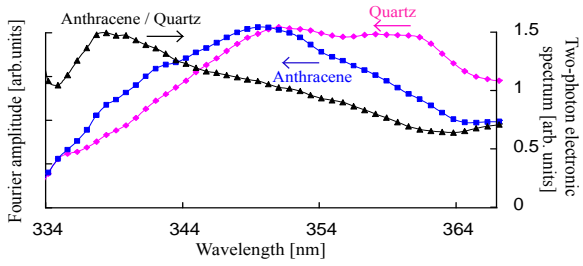

Fig. 5 FWM power spectra around a frequency of $2 \omega_{1}$ and two-photon electronic spectrum. FWM power spectra of anthracene (blue line) and quartz (red line), and two-photon electronic spectrum of anthracene (black line).

observe the TPE resonance effect. Taking advantage of the power spectrum obtained by the Fourier-transform of the FWM-IAC signal, we could identify the origin of the FWM signal. FTS technique is found to be remarkable effective tool for discriminate the origin of the FWM signal.

\section{Conclusion}

In summary, we have demonstrated FWM-FTS with 5-fs broadband pulse laser. We discussed the method for the measurement of the response function in FWM under the Raman resonance and TPE resonance, and showed that the response function could be obtained by dividing the FWM power spectrum from the resonance sample by that of the nonresonant sample. We demonstrated the origin of the FWM signal could be identified by taking advantage of the FWM power spectra obtained by the Fourier-transform of the FWM-IAC signal. In the FWM power spectrum, the Raman resonance effect and TPE resonance effect were appeared at the frequency components around frequencies of $\omega_{1}-\omega_{2}$ and $2 \omega_{1}$, respectively. The Raman spectrum over bandwidth of $3000 \mathrm{~cm}^{-1}$ was simultaneously measured. It should be noted that the FWM-FTS technique with a broadband pulse is quite effective tool for the discrimination of the origin of FWM process.

\section{References}

1) X. S. Xie, J. Yu, and W. Y. Yang: Science 312 (2006) 228.

2) X. Nan, E. O. Potma, and S. S. Xie: Biophys. J. 91 (2006) 728.

3) X. Nan, J.-X. Cheng, and X. S. Xie: J. Lipid Research, 44 (2003) 2202.

4) M. D. Duncan, J. Reintjes, and T. J. Manuccia: Opt. Lett. 7 (1982) 350 .

5) A. Zumbusch, G. R. Holtom and X. S. Xie: Phys. Rev. Lett. 82 (1999) 4142

6) K. Isobe, S. Kataoka, R. Murase, W. Watanabe, T. Higashi, S. Kawakami, S. Matsunaga, K. Fukui, and K. Itoh: Opt. Express 14 (2006) 786

7) P. M. Felker and G. V. Hartland: Chem. Phys. Lett. 134 (1987) 503.

8) M. Bellini, A. Bartoli, and T. W. Hänsch: Opt. Lett. 22 (1997) 540.

9) J. A. McGuire, W. Beck, X. Wei, and Y. R. Shen: Opt. Lett. 24 (1999) 1877

10) J. Jasapara and W. Rudolph: Opt. Lett. 24 (1999) 777. 